\title{
Inumeanuma \\ Proportion of asymptomatic AF increases after catheter ablation
}

Atrial fibrillation (AF) can be treated with catheter ablation. However, a report from the DISCERN AF study shows that, despite a reduction in the total number of AF events, the proportion of episodes that were asymptomatic increased after the procedure.

Data from implantable cardiac monitors were collected from 50 patients with symptomatic AF for 3 months before and 18 months after catheter ablation. Concurrently, patients recorded their symptoms in a diary. A total of 2,355 episodes of arrhythmia were recorded, $69 \%$ of which were AF, atrial flutter, or atrial tachycardia. Catheter ablation reduced the incidence of $\mathrm{AF}$, atrial flutter, or atrial tachycardia by $86 \%$ (from a mean of 2.0 to 0.3 episodes per day, $P<0.001$ ).

Although the presence of AF symptoms was one of the enrollment criteria, 52\% of episodes before ablation and $79 \%$ of episodes after ablation were asymptomatic. Therefore, the ratio of asymptomatic to symptomatic AF increased from 1.1 to 3.7 after the procedure. Postablation state was the strongest predictor of asymptomatic AF. Six patients (12\%) had exclusively asymptomatic $\mathrm{AF}$ after ablation. Therefore, the success rate for ablation, if defined by the absence of symptomatic AF, overestimates the actual success rate.

Changes in autonomic innervation might explain the increase in asymptomatic AF. Postablation episodes were also shorter and more likely to be atrial flutter or atrial tachycardia . Regardless, "operators should think twice before stopping chronic anticoagulation therapy in their AF patients postablation", says DISCERN AF investigator Atul Verma.

Megan Cully

Original article Verma, A. et al. Discerning the incidence of symptomatic and asymptomatic episodes of atrial fibrillation before and after catheter ablation (DISCERN AF): a prospective, multicenter study. Arch. Intern. Med. doi:10.1001/jamainternmed.2013.1561 